\title{
Processamento Implícito e Dependência Química: Teoria, Avaliação e Perspectivas
}

\author{
Ana Carolina Peuker ${ }^{1}$ \\ Fernanda Machado Lopes \\ Universidade Federal do Rio Grande do Sul \\ Carolina Baptista Menezes \\ Universidade Federal de Pelotas \\ Silvia Mendes Cunha \\ Lisiane Bizarro \\ Universidade Federal do Rio Grande do Sul
}

\begin{abstract}
RESUMO - Pesquisas recentes têm investigado mecanismos cognitivos implícitos que influenciam a decisão e o comportamento de uso da droga, como viés atencional e reatividade a pistas. Tais respostas são eliciadas automaticamente, potencializando a vulnerabilidade à dependência e recaída ao uso da droga. Este estudo teve como objetivo apresentar a perspectiva teórica dos modelos de duplo-processamento dos comportamentos aditivos assim como discutir a influência dos processos automáticos no uso de drogas, suas formas de avaliação e técnicas que objetivam modificar diretamente tais processos. Os resultados sugerem que medidas implícitas possam avaliar os mecanismos automáticos mais acuradamente do que medidas explícitas. Diante disso, sugere-se que intervenções voltadas para a transformação das cognições implícitas sejam alternativas eficazes para o tratamento da dependência química.
\end{abstract}

Palavras-chave: cognição implícita, dependência química, viés atencional

\section{Implicit Processes and Drug Addiction: Theory, Assessment and Future Directions}

\begin{abstract}
Recent studies have investigated the implicit cognitive mechanisms that influence the decision to use drugs and drug use behavior, such as attentional bias and reactivity to cues. Those responses are automatically elicited and can increase vulnerability to addiction and relapse. This review aimed to present theoretical perspective of dual-process models of addictive behaviors and to discuss the influence of automatic processes in drug intake, how they can be assessed, and techniques to directly modify them. The results suggest that implicit measures can assess the automatic mechanisms more accurately than explicit measures. Therefore, it is suggested that interventions aimed at the transformation of implicit cognitions can be effective alternatives for the treatment of addictive behaviors.
\end{abstract}

Keywords: implicit cognition, addiction, attentional bias

Teorias da adição sugerem que usuários de drogas apresentam estratégias de tomada de decisão que geralmente resultam em escolhas de recompensas imediatas apesar das possíveis consequências negativas no futuro (Verdejo-García \& Bechara, 2009; Verdejo-García, Pérez-García, \& Bechara, 2006). Dessa forma, o paradoxo central nos comportamentos aditivos é que os usuários persistem utilizando a droga mesmo (re)conhecendo os danos potenciais associados a ela. O objetivo deste artigo é apresentar e discutir a perspectiva de duplo-processamento, uma vez que esta pode contribuir para entender esse aparente paradoxo (Rooke, Hine, \& Thorsteinsson, 2008; Wiers \& Stacy, 2006). Este estudo teórico foi produzido a partir da revisão de bases de dados nacionais e internacionais (SciELO, LILACS e PUBMED) considerando como descritores de interesse as palavras dual-process models, implicit cognition, addiction e attentional bias. Foram selecionados artigos publicados nas últimas duas décadas que, dentro da temática, contemplavam um ou mais itens como: período de publicação, pertinência (aqueles que incluíam método de avaliação

1 Endereço para correspondência: Ramiro Barcelos, 2600/ sala 02 - Bairro Santa Cecília Porto Alegre, RS. CEP 90035-003. E-mail: acpeuker@hotmail.com de processos implícitos) e relevância teórica (aqueles que versavam sobre teorias de duplo processamento na dependência química e processamento implícito).

\section{Processamento Implícito e Dependência Química: Considerações Teóricas}

A maioria dos modelos de duplo processamento propõe que o comportamento humano é guiado por dois sistemas semi-independentes de processamento da informação, ainda que possa haver algumas distinções entre os níveis de análise sugeridos por diferentes modelos. Um sistema, ascendente (bottom-up) é considerado de associação rápida, não-intencional e vinculado ao afeto, em geral impulsivo, envolvendo uma avaliação automática do estímulo em termos de sua relevância motivacional e emocional. O outro sistema, descendente (top-down) é racional-analítico, intencional, incluindo processos controlados relacionados à tomada de decisão, regulação da emoção e expectativas de resultados (Rooke et al., 2008; Wiers \& Stacy, 2006).

O uso de substâncias psicoativas promove alterações em substratos neurais relacionados à emoção e à motivação. Com a exposição repetida à substância, o sistema impulsivo se tor- 
na sensibilizado aos efeitos da droga e às pistas relacionadas a ela (Robinson \& Berridge, 1993). A sensitização desses sistemas e dos seus correlatos neurais pode automatizar um conjunto de ações que levam à procura e ao uso impulsivo da droga. Isso está de acordo com o modelo da adição proposto por Tiffany (1990), que considera que os esquemas de ação associados ao uso da substância se tornam progressivamente automáticos, pois, com o uso repetido da droga, os estímulos relacionados a ela (ex.: imagens, sons, odores) passam a integrar uma rede associativa armazenada na memória.

Quando o usuário é exposto a tais pistas, os esquemas de ação são ativados, desencadeando a necessidade de consumir a substância. Dessa forma, o comportamento de uso pode iniciar e terminar sem intenção, sendo difícil de evitá-lo na presença do estímulo eliciador (Tiffany, 1990). Essa tendência de ação automatizada pode ser inibida caso o indivíduo tenha capacidade cognitiva suficiente e motivação para tal. No entanto, a inibição é menos provável entre usuários de drogas, pois estes estão mais suscetíveis e altamente sensíveis às propriedades motivacionais das pistas associadas à droga (Field \& Cox, 2008; Wiers \& Stacy, 2006).

Em termos neurobiológicos, a adição às drogas esteve predominantemente associada ao sistema cerebral de recompensa, considerado crucial para os efeitos reforçadores das drogas. Contudo, atualmente discute-se que de forma isolada o aumento dos níveis de dopamina na via mesolímbica seria insuficiente para explicar o processo aditivo como um todo; embora se admita que as mudanças estruturais e funcionais observadas na adição, incluindo alterações no córtex frontal, são mediadas pela dopamina. Devido à diminuição do processamento pré-frontal (top-down) e, portanto, das funções de controle executivo no processo de adição, há um aumento de respostas afetivas guiadas por estímulos sensoriais (bottom-up) eliciadas por pistas condicionadas aos efeitos droga (Goldstein \& Volkow, 2002).

Dessa maneira, o pobre controle sobre os impulsos e o comportamento tipicamente observados em usuários de drogas estaria relacionado ao comprometimento do funcionamento executivo (Goldstein \& Volkow, 2002; Verdejo-García et al., 2006; Verdejo-García \& Bechara, 2009; Wiers et al., 2007; Wiers \& Stacy, 2006). Esse prejuízo, aliado à saliência motivacional adquirida pelas pistas associadas à droga, podem contribuir para o aparecimento da fissura e da busca compulsiva pela substância (Field \& Cox, 2008).

Conjuntamente, todos esses aspectos refletem modificações que ocasionam um desequilíbrio entre os dois sistemas - reflexivo e impulsivo - e seus distintos circuitos neurais. O resultado é uma preponderância dos processos implícitos em detrimento de metas cognitivas explícitas (Wiers \& Stacy, 2006; Yin \& Knowlton, 2006). Dessa forma, o comportamento aditivo se torna mais impulsionado pelo estímulo e é automatizado, favorecendo a transição para o uso compulsivo da droga, à dependência e à recaída (Goldstein \& Volkow, 2002; Rooke et al., 2008; Wiers \& Stacy, 2006). Ou seja, quando um comportamento aditivo se desenvolve, o sistema de processamento emocional automático (ou "impulsivo") sofre neuroadaptações e se torna sensibilizado para os efeitos da droga e para as pistas que predizem seu uso. Algumas dessas alterações envolvem substratos neurais relacionados à emoção e à motivação. Como resultado, pistas ambientais associadas ao uso da droga capturam automaticamente a atenção do usuário, podendo eliciar um conjunto automático de tendências de ação de aproximação da droga. Essa tendência de ação pode ser inibida caso existam motivação e recursos cognitivos disponíveis para tal (processamento controlado ou "reflexivo"). Na medida em que o comportamento aditivo se desenvolve, a modulação/inibição da resposta impulsiva torna-se mais difícil. A intoxicação aguda e a exposição crônica à droga promovem o fortalecimento de tendências de aproximação automáticas e o enfraquecimento das habilidades para moderar a resposta impulsiva de uso da droga (Figura 1).

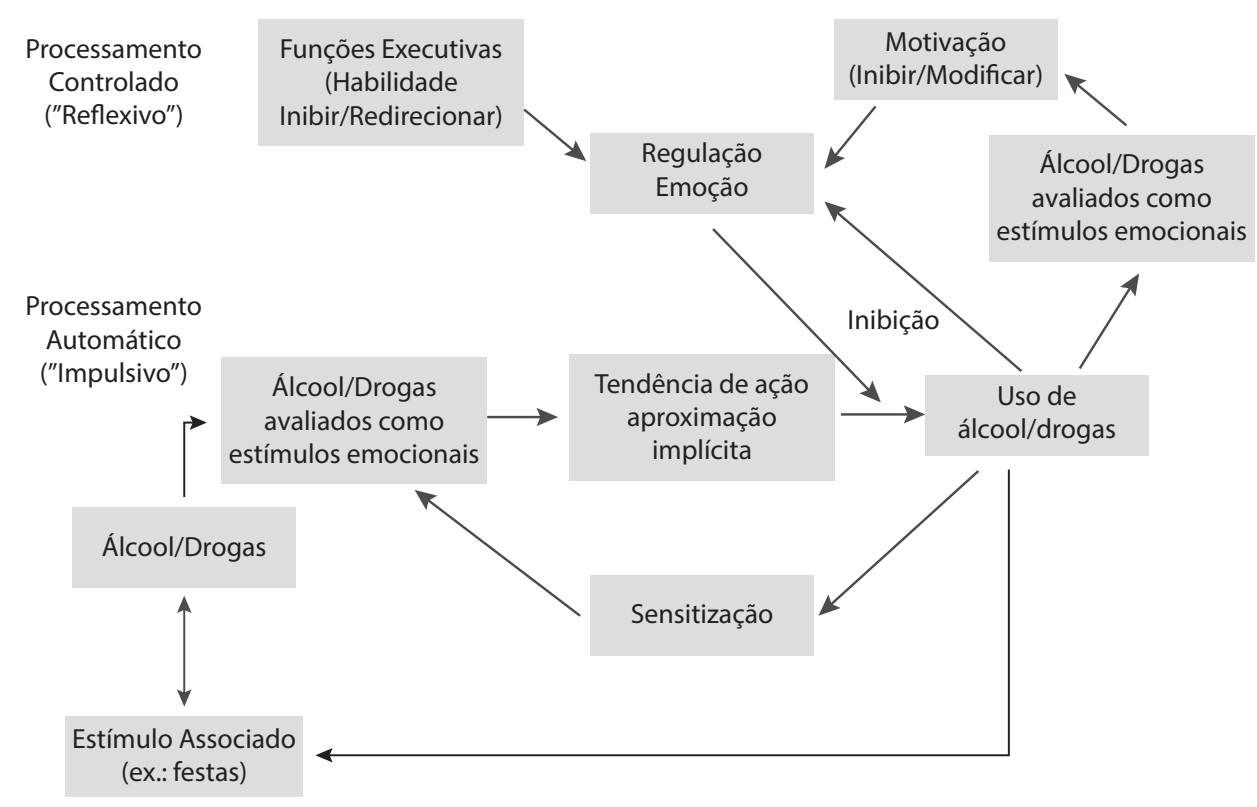

Figura 1. Visão geral esquemática dos diferentes processos envolvidos no desenvolvimento dos comportamentos aditivos (adaptado de Wiers \& Stacy, 2006). 


\section{Processamento Implícito e Dependência Química: Formas de Avaliação}

Os processos automáticos já foram identificados anteriormente na gênese dos comportamentos aditivos (Tiffany, 1990). Apesar disso, apenas recentemente um corpo de evidências tem sido formulado, à medida que novos paradigmas de avaliação dos processos automáticos ou implícitos envolvidos nas adições têm sido desenvolvidos (Wiers \& Stacy, 2006). Conforme exposto, diversos aspectos do processamento implícito, os quais independem de um processamento consciente, podem influenciar a decisão e o comportamento de uso da substância. Por isso, normalmente esse tipo de cognição é avaliada através de medidas indiretas, como o viés atencional e a reatividade a pistas (Rooke et al., 2008; Wiers \& Stacy, 2006).

O viés atencional (VA) refere-se a um processo cognitivo implícito e involuntário que pode orientar o comportamento do uso da substância em um nível pré-consciente (Cox, Fadardi, \& Pothos, 2006; Shoenmakers et al., 2010). Devido a uma predisposição natural e/ou experiências anteriores de aprendizagem, os indivíduos podem ser mais ou menos propensos a ter seu foco atencional capturado automaticamente através de pistas ambientais relacionadas à droga. Indivíduos dependentes de drogas apresentam viés cognitivo para pistas associadas a sua droga de escolha. Eles tendem a alocar sua atenção, preferencialmente, para pistas relacionadas à droga em detrimento de outros estímulos do meio. Uma vez que a atenção é capturada, tais pistas passam a exercer uma maior influência sobre o comportamento do indivíduo (Field \& Cox, 2008; Lopes, Peuker, \& Bizarro, 2008).

A tarefa de atenção visual (Visual Probe Task) é um dos principais paradigmas utilizados para investigar o VA (Peuker, Lopes, \& Bizarro, 2009). Trata-se de uma tarefa computadorizada de atenção que avalia o tempo de reação dos indivíduos para detectar e responder a um alvo apresentado onde havia um estímulo relacionado à droga. Avalia-se dessa forma a direção e as possíveis mudanças na orientação do foco atencional do participante. Por exemplo, se o participante detecta e responde mais rapidamente a estímulos que substituíam imagens relacionadas à droga do que a estímulos que substituíam imagens controle, presume-se um viés na atenção para esse tipo de estímulo. Ou seja, o VA é tido como o estado hiperatentivo do usuário de droga em direção aos estímulos condicionados a ela (Field \& Cox, 2008).

Uma vantagem que a tarefa de atenção visual oferece é poder investigar todo o processo cognitivo da atenção através da manipulação do tempo de exposição dos estímulos; desde o momento mais automático, ou implícito, até o mais explícito, ou influenciado por variáveis motivacionais e pelo esforço cognitivo. A orientação inicial é um processo relativamente rápido, mais implícito, que pode ser avaliado mesmo quando a duração da exposição dos estímulos apresentados for curta (100-200ms), indicando um viés automatizado (Field, Mogg, Zatteler, \& Bradley, 2004). Já a manutenção da atenção é um processamento mais explícito à medida que é mais influenciado por variáveis motivacionais, e pode ser medido por tempos maiores de exposição dos estímulos (2000ms). A lógica subjacente é a de que em tempos de exposição menores o indivíduo orienta sua atenção apenas uma vez para o estímulo de interesse, mas na exposição prolongada existe tempo hábil para que o indivíduo realize mais de uma mudança na orientação da atenção. Portanto, o tipo de mudança atencional capturada pela tarefa de atenção visual pode incluir a orientação inicial e, posteriormente, atenção mantida para o estímulo de interesse (Field \& Cox, 2008).

O VA pode operar de forma distinta dependendo da operação cognitiva em análise ou da população estudada. Jovens fumantes com uma história de repetidas tentativas para parar de fumar mostraram maior vigilância para imagens relacionadas ao tabaco expostas por $500 \mathrm{~ms}$ do que fumantes sem este histórico prévio de tentativas de cessação e maior viés do que não fumantes. Quando os estímulos foram apresentados por $2000 \mathrm{~ms}$ os fumantes como um todo apresentaram viés para as pistas associadas ao tabaco mas os não fumantes não, sugerindo que no tabagismo o viés estaria atrelado à manutenção da atenção (Bradley, Mogg, Wright, \& Field, 2003). Por outro lado, outros estudos com fumantes revelaram que o viés atencional pode operar durante todo o processo cognitivo da atenção (Ehrman et al., 2002; Field et al., 2004). Demonstrou-se que jovens fumantes, com dois anos e meio de uso de cigarro em média e com baixa dependência de nicotina, apresentaram viés atencional para pistas associadas à droga em todo o processo cognitivo da atenção quando comparados a não fumantes, sugerindo que fumar por poucos anos e com baixo nível de dependência pode alterar tanto a orientação inicial como a manutenção da atenção para esta classe de estímulos (Lopes, 2009).

Em outro estudo, bebedores sociais e dependentes de álcool em abstinência por mais de duas semanas apresentaram viés para pistas associados à droga no tempo de exposição $50 \mathrm{~ms}$, mas quando os estímulos foram apresentados por $500 \mathrm{~ms}$ ambos os grupos evitaram tais estímulos. Houve associação entre o viés e padrão de consumo de álcool no menor tempo de exposição, isto é, os indivíduos que bebiam maiores quantidades de álcool, na vigência do uso da droga, apresentaram maior viés. A partir disso, sugeriu-se que em tempos de exposição curtos o paradigma da tarefa de atenção visual seria útil para avaliar traço, um aspecto relacionado à gravidade da adição que não se modificaria nos períodos iniciais da abstinência. Em contraste, em tempos de exposição maiores esse paradigma avaliaria características relacionadas ao estado, que sofreriam alterações nas já primeiras semanas da abstinência (Vollstädt-Klein, Loeber, Goltz, Mann, \& Kiefer, 2009).

A avaliação direta da reatividade a pistas associadas à droga é outra abordagem que pode ser empregada nas pesquisas sobre os processos implícitos envolvidos nos comportamentos aditivos. A reatividade a pistas refere-se à variedade de respostas que são observadas quando os dependentes de drogas, ex-dependentes ou usuários frequentes são expostos ao estímulo previamente associado aos efeitos da droga (Cunha \& Bizarro, 2011; Rooke et al., 2008), tais como variáveis fisiológicas (p.ex. alterações na taxa cardíaca ou ativações neurais), subjetivas (p.ex. fissura) e comportamentais (p.ex. recaída) (Vollstädt-Klein et al., 2009). Teorias do condicionamento sugerem que a pista ambiental relacionada aos efeitos reforçadores da droga adquire valor motivacional a partir de repetidos pareamentos com os efeitos incondicionados da substância. Assim, o estímulo condicionado que 
sinaliza a disponibilidade da droga pode provocar reatividade fisiológica, psicológica e comportamental. Sendo assim, a exposição repetida ao estímulo associado à droga também pode contribuir para o comportamento de uso, provocar a busca compulsiva e a recaída em indivíduos abstinentes (Chiamulera, 2005).

Uma forma de avaliar essa reatividade é através de parâmetros psicofisiológicos, como por exemplo, a mensuração da atividade eletrocardiográfica e da resposta da condutividade da pele (Rooke et al., 2008). Respostas de reatividade a pistas têm sido utilizadas como preditores do desfecho do tratamento, envolvendo a maior predisposição à recaída. Igualmente, podem ser utilizadas como marcadores da gravidade da dependência. Através dos potenciais relacionados a evento, a análise da atividade elétrica do cérebro em resposta a imagens associadas à droga também é um campo de estudos promissor na investigação da reatividade a pistas (Polich, 2003). Estudos com imageamento cerebral também são uma alternativa, sendo que estes têm revelado correlatos neuroanatômicos da reatividade a pistas relacionadas à droga em áreas cerebrais envolvidas em processos motivacionais, emocionais e cognitivos (Chiamulera, 2005; Yalachkov, Kaiser, \& Naumer, 2011). Outra modalidade de investigação da reatividade a pistas são os estudos utilizando realidade virtual. Estudo com utilização da realidade virtual revelou que fumantes quando expostos a ambiente virtual congruente com o comportamento de fumar exibiram maiores respostas de reatividade a pistas (Traylor, Parrish, Copp, \& Bordnick, 2011).

Além da reatividade a pistas associadas à substância eliciar a fissura ou urgência em consumir a mesma, também pode ocorrer dessas pistas condicionadas às drogas induzirem a fissura por uma segunda substância. Esse fenômeno é relatado na literatura como reatividade cruzada a pistas (Cunha \& Bizarro, 2011). Por exemplo, de forma comum, a exposição a estímulos ambientais relacionados ao beber constitui um gatilho para pensamentos sobre o fumar, resultando na fissura pelo cigarro e na probabilidade aumentada de seu consumo. Tal fenômeno pode ser entendido como condicionamento de segunda ordem, ou seja, a resposta de fissura para consumo do cigarro pode ser eliciada pelas pistas associadas ao álcool e vice-versa. É possível que o consumo de álcool e as pistas relacionadas ao beber se configurem como gatilho para o comportamento de fumar, como resultado de exposições repetidas ao álcool e ao cigarro concomitantes na história de aprendizagem do indivíduo. Assim, duas respostas condicionadas são estabelecidas através de uma mesma pista: fissura para beber e para fumar (Cunha, 2010; Erblich, Montgomery, \& Bovbjerg, 2009).

O paradigma da reatividade cruzada a pistas pode justificar os elevados índices de consumo concomitante de álcool e cigarro (Cunha \& Bizarro, 2011). Tal co-ocorrência é amplamente referida pela literatura da área (Dawson, 2000; Field, Moog, \& Bradley, 2005; Hoffman, Welte, \& Barnes, 2001; Kahler et al., 2008; Mintz, Boyd, Rose, Charuvastra, \& Jarvik, 1985; White, Pandina, \& Chen, 2002). Pressupõe-se que o álcool seja um gatilho incentivo - motivacional para o uso do cigarro e, que após exposição a pistas associadas ao uso de álcool, por exemplo, há um aumento na magnitude do viés atencional para pistas associadas ao fumar (Field et al., 2005).
Metanálise realizada em estudos da área revelou que a reatividade a pistas pode gerar aumento no auto-relato de fissura e respostas fisiológicas significativas em fumantes, alcoolistas, adictos em heroína e cocaína (Carter \& Tiffany, 1999). Estudos empíricos utilizando exposição a pistas imaginárias ou in vivo relacionadas ao consumo concomitante de álcool e cigarro revelam aumento no relato de fissura por uma das substâncias após a exposição a pistas associadas a outra substância. Tal fenômeno ocorreu tanto no sentido exposição a pistas associadas ao beber eliciar fissura por cigarro como da forma inversa (Cooney et al., 2007; Drobes, 2002; Erblich et al., 2009; Palfai, Monti, Ostafin, \& Hutchison, 2000).

A exposição recorrente a pistas associadas à segunda substância pode impedir a eficácia do tratamento e a manutenção da abstinência. Por isso, é importante que essa questão seja problematizada no que se refere ao desenvolvimento de estratégias de prevenção e tratamento (Drobes, 2002). Estudo recente investigou a relação entre fissura por cigarro e fissura por crack utilizando o paradigma da exposição a pistas, no qual os pacientes foram expostos a imagens de pistas relacionadas ao consumo de crack. Após a exposição, houve aumento significativo na fissura por crack, mas também na fissura por cigarro (Araujo \& Zeni, 2011).

\section{Processamento Implícito e Comportamentos Aditivos: Intervenções Atuais}

Intervenções que visam influenciar diretamente os processos cognitivos implícitos têm sido desenvolvidas. $\mathrm{Na}$ dependência química, intervenções como o retreinamento da atenção e a meditação têm sido empregadas como técnicas complementares aos tratamentos existentes no sentido de melhorar o controle inibitório.

$\mathrm{O}$ retreinamento da atenção (attentional retraining) consiste em modificar tarefas utilizadas para avaliar o viés na atenção com o intuito de treinar a focalização da atenção para pistas não relacionadas à droga. Por exemplo, na versão original da tarefa de atenção visual, o alvo substitui imagens relacionadas à droga e imagens neutras com a mesma frequência ( $50 \%$ cada). Na versão de retreinamento, o alvo substitui as imagens neutras em $90 \%$ ou até $100 \%$ das vezes. Dessa maneira, o usuário da droga reaprende, implicitamente, a direcionar sua atenção para o estímulo neutro e a desengajar sua atenção da pista relacionada à droga. Para isso, alguns pesquisadores têm utilizado uma versão modificada da tarefa de atenção visual para manipular, mais do que para medir, o viés atencional (Field \& Eastwood, 2005; Field et al., 2007; Schoenmakers et al., 2007; Attwood, O'Sullivan, Leonards, Mackintosh, \& Munafo, 2008).

Tipicamente, nesses estudos os participantes são alocados em grupos que diferem quanto à localização dos alvos aos quais eles devem atender. Nos grupos "prestar atenção na droga", o alvo substitui imagens relacionadas à droga em todas ou na maioria das tentativas. Já nos grupos “evitar a droga", o alvo substitui imagens neutras em todas ou na maior parte das tentativas. Como os participantes são orientados a detectar e responder o mais rapidamente possível o alvo, eles devem, ao longo da tarefa, focalizar sua atenção em direção às imagens relacionadas à droga ou afastar sua atenção dessa 
classe de estímulos (Field et al., 2007; Field \& Eastwood, 2005; Schoenmakers et al., 2007).

Em um estudo com fumantes, o viés na atenção para pistas relacionadas à droga foi manipulado experimentalmente, sendo aumentado e diminuído (Attwood et al., 2008). O grupo treinado a prestar atenção ao fumar teve maiores níveis de fissura do que o grupo treinado a evitar os estímulos associados à droga. Entretanto, os resultados na fissura foram limitados aos participantes homens. Além disso, não houve efeito do retreinamento na topografia do ato de fumar (número de tragadas, volume da tragada, etc.). Embora haja evidência de correlação positiva entre o viés na atenção e a fissura, ainda se faz necessário comparar o retreinamento da atenção com uma condição controle na qual o viés não tivesse sido manipulado (Attwood et al., 2008).

Fumantes treinados para atender às pistas associadas à droga apresentaram maior viés após o retreinamento, enquanto os treinados a evitar tais estímulos em apenas uma sessão tiveram decréscimo no viés em relação à linha de base. Já no grupo controle, no qual o viés não foi manipulado, não foram observadas modificações. Os efeitos de uma sessão isolada foram considerados pelos autores como uma modificação específica no desempenho da tarefa, mais do que uma mudança global no viés atencional para pistas relacionadas à droga, pois a modificação do viés atencional não produziu efeitos generalizáveis (Field, Duka, Tyler, \& Schoenmakers, 2009). Por isso, ainda é necessário elucidar se efeitos similares podem ser observados em fumantes após múltiplas sessões de modificação e se há generalização para outras medidas de viés atencional.

Outra forma de treinamento da atenção que vem sendo crescentemente investigada é a meditação. Considerada uma espécie de treinamento mental, essa prática pode ser dividida em dois tipos principais, conforme o direcionamento da atenção. Na meditação concentrativa, considerada treinamento do foco fechado, o praticante focaliza sua atenção sobre um único objeto - respiração, contagem, imagem, entre outros - de forma sistemática e contínua. O objetivo dessa prática é aumentar a capacidade de controle sobre a concentração e a capacidade de não deixar distrair-se com os diversos estímulos que chegam à percepção durante o processo meditativo. $\mathrm{Na}$ meditação de atenção plena (mindfulness), considerada treinamento do monitoramento aberto, o praticante mantém sua atenção alerta e vigilante, observando todos os estímulos que chegam à sua consciência, porém de forma desapegada (dettached mindfluness), isto é, não analítica, sem qualquer tipo de julgamento ou elaboração a respeito dos mesmos (Lutz, Slagter, Dunne, \& Davidson, 2008).

Apesar de possuírem tipos de alocação atencional distintos, a meditação concentrativa e a meditação de atenção plena possuem objetivos e características comuns. Através do exercício da auto-observação e do treinamento da concentração, ambas visam à ampliação da autoconsciência e da consciência do momento presente. Por meio dessa consciência e do controle atencional, busca-se reduzir a identificação com processos mentais automatizados, os quais são ou estão, muitas vezes, disfuncionais. Além disso, busca-se diminuir a suscetibilidade a distrações oriundas de estímulos externos, sensoriais, e estímulos internos, como os próprios pensamentos e emoções. Por isso, e conforme tem sido de- monstrado, a meditação pode ser entendida, na perspectiva da ciência psicológica, em especial da psicologia cognitiva e da neurociência cognitiva, como uma técnica autorregulatória e metacognitiva (Lutz, Dunne, \& Davidson, 2007).

Avaliações de meditadores através de tarefas que investigam a autorregulação demonstram que o praticante adquire uma maior capacidade de controlar de forma voluntária, ou seja, não automatizada, o direcionamento de seus recursos mentais e, consequentemente, o seu comportamento (Lutz et al., 2008; Tang et al., 2007). Além disso, a meditação tem sido associada a uma menor reatividade psicofisiológica (Tang \& Posner, 2009), à redução de pensamentos ruminativos (Jain et al., 2007) e a uma maior concordância entre a experiência afetiva explícita e implícita (Brown \& Ryan, 2003). Tomados em conjunto, esses dados sugerem que a meditação de fato parece influenciar a capacidade do praticante controlar seus processos mentais e comportamentos e ampliar a consciência dos mesmos.

Com relação à cessação do uso de drogas, o efeito de intervenções utilizando a meditação como uma de suas ferramentas tem sido testado empiricamente. Após utilizar um programa de intervenção com meditação de oito semanas - o Programa de Redução de Estresse Baseado em Mindfulness (Mindfulness-Based Stress Reduction Program -MBSR) para fumantes, demonstrou-se que $56 \%$ dos participantes atingiram a abstinência. Esse resultado se manteve após avaliação de seguimento de seis semanas (Davis, Fleming, Bonus, \& Baker, 2007). Um dos efeitos da meditação na redução do uso de drogas é o decréscimo da tentativa de evitar pensamentos indesejáveis com relação às mesmas. Em outras palavras, a maneira com que o usuário reage a seus conteúdos mentais parece ser mais importante do que a supressão dos mesmos (Bowen, Witkiewitz, Dillworth, \& Marlatt, 2007). Esses resultados são relevantes no que concerne ao processamento implícito e a sua relação com o abuso de drogas, uma vez que um maior controle sobre a atenção e sobre a reatividade aos processos mentais pode ser crucial para que o usuário consiga interromper o fluxo de associações aprendidas que o impulsionam a buscar e a consumir a substância.

Atualmente existe uma adaptação do MBSR, chamada de Programa de Prevenção de Recaída Baseado em Mindfulness (Mindfulness-Based Relapse Prevention Program-MBRP), que visa atender especificamente às necessidades e dificuldades dos usuários de substâncias (Bowen et al., 2009). Essa versão integra atividades do tratamento padrão de prevenção de recaída, técnicas da terapia cognitivo-comportamental, assim como práticas de meditação e relaxamento adaptadas a esta população - p. ex., duração de 20 a 30 minutos, ao invés de 45. A adaptação do programa foi testada com usuários de álcool (45.2\%), cocaína/crack (36.2\%), metaanfetamina (13.7\%), opióide/heroína (7.1\%), maconha (5.4\%) e outros (1.9\%). Em comparação ao tratamento usual, o MBRP produziu significativa redução no uso dessas substâncias e na fissura. Além disso, em uma escala de satisfação de 0 a 10, a média da avaliação foi de 8.3 , sendo que no seguimento de dois meses, $86 \%$ relataram a continuidade da aplicação das técnicas. Um estudo subsequente (Witkiewitz, Bowen, Douglas, \& Hsu, 2012) apontou que a redução da fissura foi mediada pelo aumento dos escores de aceitação e não julga- 
mento, os quais foram medidos pela Escala de Competência e Adesão ao MBRP (Chawla et al., 2010). Esse dado apoia a eficácia do MBRP, já que essas são justamente as qualidades que o mesmo visa desenvolver.

Portanto, parece que a meditação e o relaxamento podem ser uma alternativa para o tratamento complementar de dependentes químicos, sem transtorno psicótico ou risco de suicídio, já que estes foram excluídos do estudo. É importante destacar que nesse programa solicita-se que os participantes incorporem as qualidades cultivadas pelas práticas, tal como maior atenção e consciência, nas suas atividades diárias, o que poderia facilitar a adesão e eficácia das mesmas. Além disso, ressalta-se que essas intervenções são coordenadas e conduzidas por profissionais da área da saúde com treinamento tanto no tratamento usual, como no uso das técnicas complementares.

Em suma, o treino em meditação parece ser uma complementação para o tratamento do uso de drogas, uma vez que estimula habilidades como maior consciência das ações, menor reatividade aos estímulos e o uso de estratégias autorregulatórias mais eficazes, tais como o controle da alocação da atenção e inibitório. No que tange ao duplo-processamento, essas habilidades podem ser especialmente úteis para que o usuário consiga modular as respostas impulsivas, facilitando a expressão de comportamentos guiados pelo sistema reflexivo.

\section{Discussão}

No contexto da dependência química, as teorias de duplo-processamento auxiliam na compreensão de como os estímulos relacionados à droga são processados e de que forma o desequilíbrio entre os processos automáticos e controlados desencadeia sua utilização compulsiva. Reações fisiológicas, emocionais e comportamentais provocadas automaticamente pela droga ou pista associada decorrem de uma rede associativa que se auto-regula. Essa atividade, também descrita como ascendente, é mediada por estruturas sub-corticais mais primitivas que favorecem a ação impulsiva. Além de apresentar esse tipo de processamento, sua relevância para a investigação no campo dos transtornos aditivos e algumas formas de avaliá-lo, discutiu-se como se pode intervir na inter-relação entre os sistemas controlados e automáticos através de estratégias regulatórias oriundas do sistema reflexivo, ou descendente. As atividades desse tipo de processamento são mediadas por estruturas corticais superiores e envolvem habilidades de atenção executiva, tais como controle da alocação de recursos mentais, auto-monitoramento, resolução de conflitos e controle inibitório.

Em termos neurais, esses dois tipos de processamento dependem tanto de estruturas comuns, como de redes neurais distintas. Ou seja, embora possuam mecanismos separados, esses sistemas se relacionam (Ochsner et al., 2009). Dessa forma, devido às suas interações, é possível modular o processamento automático (ascendente) e aumentar o controle da resposta impulsiva através de habilidades superiores (descendente).

Discutiu-se neste estudo a influência dos processos automáticos no uso de drogas, suas formas de avaliação e técnicas que podem ser empregadas na modificação direta desses processos, como os treinos de reorientação da atenção e meditação. A dependência de substâncias psicoativas é um transtorno que provoca uma variedade de efeitos e fenômenos que decorrem da interação de fatores farmacológicos, psicológicos e ambientais. Como consequência disso, são produzidas alterações em áreas cerebrais relacionadas à motivação e às emoções que levam a padrões compulsivos e estereotipados de consumo. Além disso, a adição pode ser mediada por processos cognitivos implícitos, que ocorrem independentemente do conhecimento dos efeitos prejudiciais da substância (Chiamulera, 2005; Rooke et al., 2008; Wiers \& Stacy, 2006).

A área de pesquisa sobre novas intervenções que modifiquem processos cognitivos implícitos e auxiliem os usuários de drogas a exercer maior controle executivo sobre tais processos parece promissora. No que concerne à sua investigação e identificação, comparadas às medidas explícitas, as avaliações da cognição implícita são menos sensíveis aos efeitos de desejabilidade social e podem auxiliar no entendimento das circunstâncias nas quais o comportamento de um indivíduo é incongruente com metas explícitas mantidas por ele. Contudo, os instrumentos de mensuração ainda estão sendo elaborados. Por isso, tais medidas ainda precisam ser testadas, melhoradas e outras ferramentas devem ser desenvolvidas. Também são necessários estudos de validade e confiabilidade de tarefas que avaliam o processamento implícito, como o viés atencional e a reatividade a pistas. Além disso, essas medidas precisam ser relacionadas a outros indicadores de severidade clínica, pois poucos estudos mostraram relações entre elas e avaliações de resultados clínicos (Lopes et al., 2008; Wiers \& Stacy, 2006).

Através destas avaliações deve-se buscar compreender o quanto os sistemas de processamento implícito (automático) e explícito (reflexivo) interagem um com outro para influenciar a tomada de decisão e o comportamento, tanto na condição de adição à droga como após intervenções. Da mesma forma, é necessário investigar sob que circunstâncias, especialmente considerando diferenças individuais, grupais e culturais, um tipo de processamento se sobrepõe mais facilmente ao outro e de que maneira os conflitos entre esses sistemas podem ser solucionados. No entanto, essas proposições ainda necessitam maior confirmação empírica. Se validadas, a lacuna existente entre os modelos neurobiológicos da adição, amplamente baseados na pesquisa experimental com animais, e a pesquisa sobre a psicologia dos comportamentos aditivos em humanos poderá ser preenchida.

\section{Referências}

Araujo, R. B., \& Zeni, T. C. (2011). Relação entre o craving por tabaco e o craving por crack em pacientes internados para desintoxicação. Jornal Brasileiro de Psiquiatria, 60, 28-33.

Attwood , A. S., O’Sullivan, H., Leonards, U., Mackintosh, B., \& Munafo, M. R. (2008). Attentional bias training and cue reactivity in cigarette smokers. Addiction 103, 1875-1882.

Bowen, S., Chawla, N., Collins, S. E., Witkiewitz, K., Hsu, S., Grow, J., ...Marlatt, A. (2009). Mindfulness-based relapse prevention for substance use disorders: A pilot efficacy trial. Substance Abuse, 30, 295-305. 
Bowen, S., Witkiewitz, K., Dillworth, T. M., \& Marlatt, G. A. (2007). The role of thought suppression in the relationship between mindfulness meditation and alcohol use. Addictive Behaviors, 32, 2324-2328.

Brown, K. W., \& Ryan, R. M. (2003). The benefits of being present: mindfulness and Its role in psychological well-being. Journal of Personality and Social Psychology, 84, 822-848.

Bradley, B. P., Mogg, K., Wright, T., \& Field, M. (2003). Attentional bias in drug dependence: vigilance for cigaretterelated cues in smokers. Psychology of Addictive Behaviors, 17, 66-72.

Carter, B. L., \& Tiffany, S. T. (1999). Meta-analysis of cue reactivity in addiction research. Addiction, 94, 327-340.

Chawla, N., Collins, S., Bowen, S., Hsu, S., Grow, J., Douglas, A., \& Marlatt, G. A. (2010). The Mindfulness-Based Relapse Prevention Adherence and Competence Scale: Development, Interrater Reliability and Validity. Psychotherapy Research, 20(4), 388-397.

Chiamulera, C. (2005). Cue reactivity in nicotine and tobacco dependence: a "multiple-action" model of nicotine as a primary reinforcement and as an enhancer of effects of smoking-associated stimuli. Brain Research Reviews, 48, 74-97.

Cooney, N., Litt, M., Cooney, J., Pilkey, D., Steinberg, H., \& Oncken, C. A. (2007). Alcohol and Tobacco Cessation in Alcohol-Dependent Smokers: Analysis of Real-Time-Reports. Psychology of Addictive Behaviors, 21, 277-286.

Cox, W. M., Fadardi, J. S., \& Pothos, E. M. (2006). The addictionstroop test: Theoretical considerations and procedural recommendations. Psychological Bulletin, 132, 443-76.

Cunha, S. M. (2010). Viés atencional para o cigarro em bebedores jovens fumantes após exposição a imagens relacionadas ao álcool. Dissertação de Mestrado, Universidade Federal do Rio Grande do Sul. Porto Alegre.

Cunha, S. M., \& Bizarro, L. (2011). Reatividade cruzada a pistas no consumo de álcool e cigarro: Revisão crítica da literatura. Interação em Psicologia, 15, 121-128.

Davis, J. M., Fleming, M. F., Bonus, K. A., \& Baker, T. B. (2007). A pilot study on mindfulness based stress reduction for smokers. BMC Complementary and Alternative Medicine, 7, 1-7.

Dawson, D. (2000). Drinkink as a risk factor for sustained smoking. Drug and Alcohol Dependence, 59, 235-249.

Drobes, D. J. (2002). Cue reactivity in alcohol and tobacco dependence. Alcoholism: Clinical and Experimental Research, 26(12), 1928-1929.

Ehrman, R. N., Robbins, S. J., Bromwell, M. A., Lankford, M. E., Monterosso, J. R., \& O’Brien, C. P. (2002). Comparing attentional bias to smoking cues in current smokers, former smokers, and non-smokers using a dot-probe task. Drug and Alcohol Dependence, 67, 185-191.

Erblich, J, Montgomery, G. H., \& Bovbjerg, D. H. (2009). Scriptguided imagery of social drinking induces both alcohol and cigarette fissura in a sample of nicotine-dependent smokers. Addictive Behaviors, 34,164-70.

Field, M., \& Cox, W. M. (2008). Attentional bias in addictive behaviors: a review of its development, causes, and consequences. Drug and Alcohol Dependence, 1, 1-20.

Field, M., Duka, T., Tyler, E., \& Schoenmakers, T. (2009). Attentional bias modification in tobacco smokers. Nicotine Tobacco Research, 11, 812-22
Field, M., \& Eastwood, B. (2005). Experimental manipulation of attentional bias increases the motivation to drink alcohol. Psychopharmacology, 183, 350-357.

Field, M., Mogg, K., \& Bradley, B. (2005). Alcohol increases cognitive biases for smoking cues in smokers. Psychopharmacology, 180, 63-72.

Field, M., Mogg, K., Zatteler, J., \& Bradley, B. (2004). Attentional biases for alcohol cues in heavy and light social drinkers: The roles of initial orienting and maintained attention. Psychopharmacology, 176, 88-93.

Field, M., Duka, T., Eastwood, B., Child, R., Santarcangelo, M., \& Gayton, M. (2007). Experimental manipulation of attentional bias in heavy drinkers: do the effects generalize? Psychopharmacology, 192, 593-608.

Goldstein, R. Z., \& Volkow, N. D. (2002). Drug addiction and its underlying neurobiological basis: neuroimaging evidence for the involvement of the frontal cortex. American Journal of Psychiatric, 159, 1643-1652.

Hoffman, J., Welte, J., \& Barnes, G. (2001). Co-occurrence of alcohol and cigarette use among adolescents. Addictive Behaviors, 26, 63-78.

Kahler, C., Strong, D., Papandonatos, G., Colby, S., Clark, M., Boerges, J., ...Buka, S. (2008). Cigarette smoking and the lifetime alcohol involvement continuum. Drug and Alcohol Dependence, 93, 111-120.

Jain, S., Shapiro, S. L., Swanick, S., Roesch, S. C., Mills, P. J., Bell, I., \& Schwartz, G. E. (2007). A randomized controlled trial of mindfulness meditation versus relaxation training: effects on distress, positive states of mind, rumination and distraction. Annals of Behavioral Medicine, 33, 11-21.

Lopes, F. M. (2009). Viés atencional em jovens fumantes. Dissertação de Mestrado. Programa de Pós-graduação em Psicologia, Instituto de Psicologia, Universidade Federal do Rio Grande do Sul. Porto Alegre, Brasil.

Lopes, F. M., Peuker, A. C., \& Bizarro, L. A. (2008). Viés atencional em fumantes. Psico, 39, 280-288.

Lutz, A., Dunne, J. D., \& Davidson, R. J. (2007). Meditation and the neuroscience of consciousness: An introduction. In P. Zelazo, M. Moscovitch \& E. Thompson (Eds.), Cambridge Handbook of Consciousness (pp. 499-554). New York: Cambridge University Press.

Lutz, A., Slagter, H. A., Dunne, J. D., \& Davidson, R. J. (2008). Attention regulation and monitoring in meditation. Trends in Cognitive Sciences, 12, 163-169.

Mintz, J., Boyd, G., Rose, J., Charuvastra, V., \& Jarvik, M. (1985). Alcohol increases cigarette smoking: A laboratory demonstration. Addictive Behaviors, 10, 203-207.

Ochsner, K. N., Ray, R. R., Hughes, B., McRae, K., Cooper, J. C., Weber, J., ...Gross, J. (2009). Bottom-up and top-down processes in emotion generation: Common and distinct neural mechanisms. Psychological Science, 20, 1322-1331.

Palfai, T. P., Monti, P. M., Ostafin, B., \& Hutchison, K. (2000). Effects of nicotine deprivation on alcohol related information processing and drinking behavior. Journal of Abnormal Psychology, 109, 96-105.

Peuker, A. C., Lopes, F. M., \& Bizarro, L. (2009). Viés atencional no abuso de drogas: teoria e método. Psicologia: Teoria e Pesquisa, 25, 505-512.

Polich, J. (2003). Event-related potentials and everyday drugs. Brain and Cognition, 53, 45. 
Robinson, T. E., \& Berridge, K. C. (1993). The neural basis of drug fissura: An incentive-sensitization theory of addiction. Brain Research Reviews, 18, 247-291.

Rooke, S. E., Hine, D. W., \& Thorsteinsson, E. B. (2008). Implicit cognition and substance use: a meta-analysis. Addictive Behaviors, 33, 1314-28.

Schoenmakers, T., Bruin, M., Lux, I., Goertz, A., Van Kerkhof, D., \& Wiers, R. (2010). Clinical effectiveness of attentional bias modification training in abstinent alcoholic patients. Drug and Alcohol Dependence, 109, 30-36.

Schoenmakers, T., Wiers, R., Jones, B., Bruce, G., \& Jansen, A. (2007). Attentional re-training decreases attentional bias in heavy drinkers without generalization. Addiction, 102, 399405.

Tang, Y., Ma, Y., Wang, J., Fan, Y., Feng, S., Lu, Q., ...Posner, M. (2007). Short-term meditation training improves attention and self-regulation. Proceedings of the National Academy of Sciences, 104, 17152-17156.

Tang, Y., \& Posner, M. I. (2009). Attention training and attention state training. Trends in Cognitive Sciences, 13, 222-227.

Tiffany, S. (1990). A cognitive model of drug urges and drug-use behavior: Role of automatic and non-automatic processes. Psychological Review, 97, 147-168.

Traylor, A., Parrish, D., Copp, H., \& Bordnick, P. (2011). Using virtual reality to investigate complex and contextual cue reactivity in nicotine dependent problem drinking. Addictive Behaviors, 36, 1068-1075.

Verdejo-García, A., \& Bechara A. (2009). A somatic marker theory of addiction. Neuropharmacology, 56, 48-62.

Verdejo-García A., Pérez-García, M., \& Bechara,A. (2006). Emotion, decision-making and substance dependence: a somatic-marker model of addiction. Current Neuropharmacology, 4, 17-31.
Vollstädt-Klein, S., Loeber, S., von der Goltz, C., Mann, K., \& Kiefer, F. (2009). Avoidance of alcohol-related stimuli increases during the early stage of abstinence in alcoholdependent patients. Alcohol and Alcoholism, 44, 458-63.

Wiers, R. W., Bartholow, B. D., van der Wildenberg, E., Trush, C., Engels, R. C., Sher, K. J., ...Stacy, A. W. (2007). Automatic and controlled process and the development of addictive behaviors in adolescents: a review and a model. Pharmacology Biochemistry and Behaviors, 86, 263-283.

White, H., Pandina, R., \& Chen, P. (2002). Developmental trajectories of cigarette use from early adolescence into young adulthood. Drug and Alcohol Dependence, 65, 167-178.

Wiers, R., \& Stacy, A. (2006). Implicit cognition and addiction. Current Directions in Psychological Science, 15, 292-296.

Witkiewitz, K., Bowen, S., Douglas, H., \& Hsu, S.H. (2012). Mindfulness-based relapse prevention for substance craving. Addictive Behaviors, 38(2), 1563-1571. doi: 10.1016/j. addbeh.2012.04.001.

Yalachkov,Y., Kaiser, J., \& Naumer, M. (2011). Functional neuroimaging studies in addiction multisensory drug stimuli and neural cue reactivity. Neuroscience \& Behavioral Reviews, $36,825-835$.

Yin, H. H., \& Knowlton, B. J. (2006). Addiction and learning in the brain. In R. Wiers \& A. W. Stacy (Eds.), Handbook of Implicit Cognition and Addiction (pp. 185-199). Thousand Oaks, CA: Sage.

Recebido em 23.12.2010

Primeira decisão editorial em 22.05.2012

Versão final em 06.07.2012

Aceito em 09.07.2012 